\title{
253 PD1 AND LAG3 CONVERGE TO LIMIT POLYFUNCTIONALITY AND SYSTEMIC IMMUNITY
}

${ }^{1}$ Lawrence Andrews*, ${ }^{2}$ Sasikanth Manne, ${ }^{2} E$ John Wherry, ${ }^{1}$ Creg Workman, ${ }^{1}$ Dario Vignali. ${ }^{1}$ University of Pittsburgh, Pittsburgh, USA; ${ }^{2}$ University of Pennsylvania, Philadelphia, PA, USA

Background Targeting PD1 with monoclonal antibodies has yielded clinical success across a variety of tumor types, yet overcoming inhibitory receptor (IR)-mediated tolerance is essential to improve immunotherapeutic responses. LAG3 coexpresses with PD1 on CD8 + tumor-infiltrating T cells (TIL), signifying a highly exhausted phenotype, and dual PD1/LAG3 blockade in $\mathrm{C} 57 \mathrm{BL} / 6$ mice enhances antitumor immunity. As CD8 + TIL is the dominant LAG3-expressing TIL population, it is hypothesized that PD1 and LAG3 synergizes to limit CD8 + TIL function controlling antitumor immunity.

Methods To understand the cellular and mechanistic basis for PD1/LAG3 synergy, conditional knock-in mice "surgically dissect" Pdcd1 and/or Lag3 floxed alleles restricted to CD8 + T cells expressing E8ICre.GFP. These mice were crossed with the Pmel transgene to assess PD1 and/or LAG3-sufficient or deficient gp100-specific CD8 $+\mathrm{T}$ cell populations. CD8+ Pmel cells were isolated and adoptively transferred into C57BL/6 mice harboring a B16-gp100-overexpressing tumor to observe therapeutic benefit, or to assess $\mathrm{T}$ cell functionality within the tumor.

Results While little therapeutic benefit was observed with a prophylactic adoptive transfer of wild-type CD8+ Pmel cells into mice which then received B16-gp100 tumor, there was reduced tumor growth in mice receiving PD1-deficient CD8+ Pmel cells, which was further enhanced in mice receiving PD1/LAG3-deficient CD8 + Pmel cells with long-term tumorfree survival. Likewise, a therapeutic administration of PD1/ LAG3-deficient CD8 + Pmel cells into mice once the tumor has been established showed an initial therapeutic benefit, with enhanced survival, that was not demonstrated with adoptive transfer of PD1 or LAG3-deficient, or wild-type, counterparts. Each PD1 and/or LAG3-sufficient or deficient CD8+ Pmel mice were differentially congenically marked to assess each of the four genotypes that can be adoptively transferred into the same host as a "quad transfer" system. Recovery of these populations within the tumor show that the PD1/LAG3deficient CD8 + Pmel cells out-compete PD1 or LAG3-deficient, or wild-type, counterparts due to enhanced proliferation (Ki67/BrdU). Furthermore, PD1/LAG3-deficient CD8 + T cells were more functional with increased IFNg and GzmB release observed by flow cytometry.

Conclusions Overall PD1 and LAG3 limit anti-tumor immune effects as removal of both IRs on a gp100 antigen-specific $\mathrm{CD} 8+\mathrm{T}$ cell population results in reduced B16-gp100 tumor growth and enhanced survival in an adoptive transfer model, as a result of enhanced CD8 + TIL functionality and proliferation. These results provide striking evidence that the development of anti-LAG3 agents in the clinic would yield improved responses with anti-PD1.

http://dx.doi.org/10.1136/jitc-2021-SITC2021.253 